\title{
Tools for accurate assessment of botrytis bunch rot (Botrytis cinerea) on wine grapes
}

\author{
G.N. Hill ${ }^{1}$, R.M. Beresford ${ }^{1}$ and K.J. Evans ${ }^{2}$ \\ ${ }^{1}$ The New Zealand Institute for Plant E Food Research Limited (Plant E Food Research), \\ Mt Albert Research Centre, Private Bag 92169, Auckland 1142, New Zealand \\ ${ }^{2}$ Perennial Horticulture Centre, Tasmanian Institute of Agricultural Research, University \\ of Tasmania, \\ 13 St Johns Avenue, New Town, TAS 7008, Australia \\ Corresponding author: gareth.hill@plantandfood.co.nz
}

\begin{abstract}
Botrytis bunch rot (botrytis) can reduce grape yield and wine quality. Standardised assessment methods are needed to allow greater precision in wine making and to allow the use of disease management decision support models in grape production. This study developed a botrytis disease assessment key to assist the accuracy of visual disease assessment. Associated computer training software, designed to improve the accuracy of disease assessments, was also developed and tested. The mean absolute error in estimates of percentage botrytis severity was significantly $(\mathrm{P}<0.05)$ lower after eight assessors used the training software. Vineyard sample size required for estimation of disease severity was determined and a disease incidence-severity relationship for estimating severity from incidence was developed.
\end{abstract}

Keywords assessor training, disease assessment, incidence-severity relationship, variation, botrytis bunch rot.

\section{INTRODUCTION}

Botrytis bunch rot (botrytis), caused by Botrytis cinerea, can decrease grape yield and wine quality (Nair \& Hill 1992) and wineries often impose price penalties if more than 3-5\% of the harvested crop is affected by botrytis. Accurate quantification of botrytis in the vineyard is therefore important to both growers and wine makers. In addition, the use of decision support models to help manage botrytis risk is increasing and these models require high quality disease data from standardised methods in order to make accurate predictions. There is currently a lack of standardisation in botrytis assessment methods in the New Zealand and Australian wine industries.

In general, disease can be measured either as incidence or severity (Seem 1984). Disease incidence is the number of plant parts that are diseased out of all those sampled, e.g. the proportion of grape bunches that are diseased. Disease severity is the intensity of disease affecting plant parts, e.g. the proportion of diseased grape berries in a bunch. Disease severity is the variable of most interest to grape growers and wine makers because it better reflects the impact of botrytis on yield and 
wine making than incidence. Botrytis severity is recorded in the New Zealand and Australian Wine industries as a percentage on individual bunches and the average severity of a sample is used to describe the amount of botrytis present in the vineyard. Average severity is sometimes referred to by terms such as botrytis crop loss or percentage crop infection, but this paper uses the term mean botrytis severity.

There is often a close relationship between incidence and severity, at least for low severities, and such a relationship can be used to estimate severity from incidence (Seem 1984). Accuracy and precision (repeatability) of disease severity assessment can be improved though the use of assessment keys and assessment training methods (James 1974; Nutter et al. 2006).

The aim of this study was to develop methods for improving the accuracy, repeatability and speed of botrytis assessment in grapevines. A botrytis disease assessment key and assessment training software, called Bunch Rot Assessment Trainer (BRAT), were developed using principles described by Nutter et al. (2006). A disease incidence-severity relationship was also developed to allow rapid estimation of botrytis severity from low incidence to assist quantification of botrytis early in the development of an epidemic.

\section{MATERIALS AND METHODS}

\section{Visual assessment of botrytis severity}

Botrytis severity was estimated on individual grape bunches by estimating the percentage of berries or tissue exhibiting botrytis symptoms for each bunch. Symptoms were recorded as botrytis only if $B$. cinerea sporulation was present within the rotting part of the bunch. Rot symptoms not attributable to $B$. cinerea were recorded separately. Mean botrytis severity $(S)$ for all bunches assessed was calculated as follows:

$$
S=\sum S_{i} / n, \quad \text { Eq. (1) }
$$

where $S_{i}=\%$ severity for the $i^{t h}$ bunch and $n=$ the total number of bunches assessed.

\section{Botrytis severity assessment key}

A digital photograph of a healthy grape bunch was stylised using image manipulation software (GNU Image Manipulation Program; http://gimp. org). Certain areas of the image were shaded to represent varying percentages of botrytis severity according to a typical pattern of botrytis spread on grape bunches in the vineyard. Image analysis was used to determine the exact percentage of the shaded area to within $\pm 1 \%$ (Figure 1).

\section{Development and evaluation of BRAT}

Software was developed using the programming language Microsft ${ }^{\mathrm{TM}} \mathrm{C} \#$ to improve the accuracy and precision of botrytis estimates made by assessors. Diseased bunch images were created in the same manner as for the assessment key. The software was tested by eight scientific staff from Plant \& Food Research. For each training session, each user was presented with 20 diseased bunch images, randomly selected from 81 possible images, and the user entered a severity estimate for each image. At the end of each session the user was shown a graph of their estimated severity values plotted against actual severity. A fitted regression line was shown on the graph, as well as the equation and coefficient of determination $\left(\mathrm{R}^{2}\right)$ for that line. Results from three sessions for each user (1. pre-training, 2. with assessment key and 3. posttraining without assessment key) were analysed using the regression slope and intercept and mean error (ME) to compare accuracy and $\mathrm{R}^{2}$ and mean absolute error (MAE) to compare precision. $\mathrm{ME}$ and MAE were calculated as follows:

$$
\begin{array}{ll}
\mathrm{ME}=\Sigma\left(S_{a}-S_{e}\right) / n, & \text { Eq. (2) } \\
\mathrm{MAE}=\sum\left|\left(S_{a}-S_{e}\right)\right| / n, & \text { Eq. (3) }
\end{array}
$$

where $S_{a}=$ actual severity of each image, $S_{e}=$ estimated severity of each image and $n=$ the number of images assessed.

\section{Sample size}

To determine the number of bunches required to obtain an estimate of disease severity of known precision, a method similar to that described by Kranz (1988) was used. Botrytis severity data 

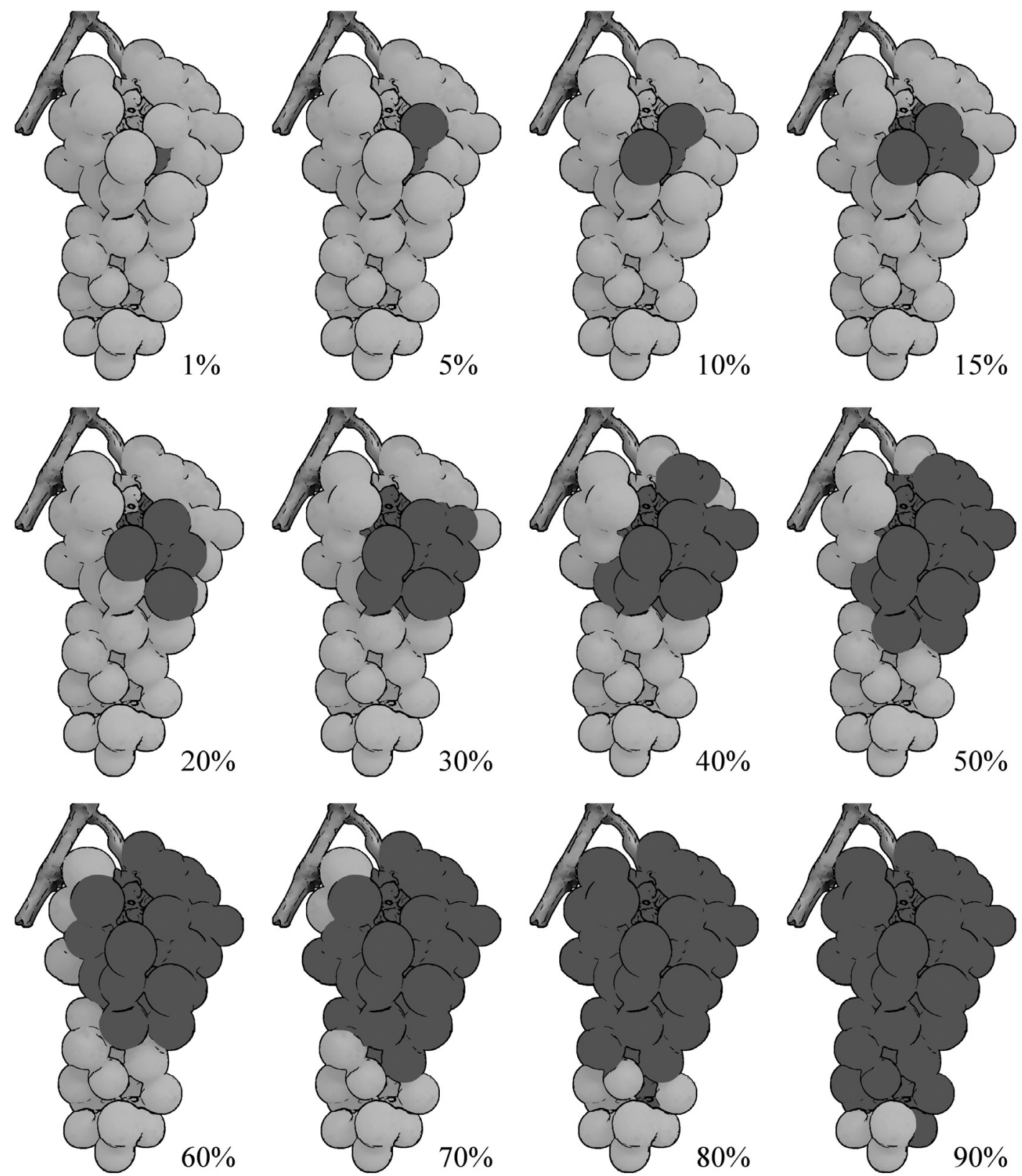

Figure 1 Botrytis bunch rot severity assessment key. Lighter areas represent healthy berries; darker areas represent diseased berries. Numbers indicate the percentage of the visible side of the bunch occupied by diseased berries (image analysis software was used to calculate percentages to within $\pm 1 \%$ ). 
from replicated small-plot trials in five vineyards, where 150-250 bunches had been visually assessed in the 2008/2009 season, were used to select random subsets of increasing sample size, ranging from two bunches to all available bunches. The coefficient of variation $(\mathrm{CV})$ was calculated for each of these subsets. CV was plotted against sample size and the minimum sample size at which the mean of repeated samples was within $5 \%$ of the CV for all bunches was determined. This was repeated ten times for each vineyard with the data being randomised before each repetition. The results of the ten repetitions for each sample size were pooled for all vineyards and a mean minimum sample size was calculated.

\section{Incidence-severity relationship}

An incidence-severity (I-S) relationship was determined using botrytis disease data from 22 replicated disease management trials conducted between 2006 and 2009 in vineyards in Hawke's Bay, Marlborough and Pukekohe in New Zealand and Tasmania and Victoria in Australia. A total of 126 pairs of values of mean botrytis severity and percentage botrytis incidence at harvest were derived from samples of 30-50 bunches per experimental plot (4-7 vines). Percentage severity $(S)$ values were transformed to logit severity $\left(S_{\text {logit }}\right)$ :

$$
S_{\text {logit }}=\ln ((S+0.1) /(100.1-S)) \quad \text { Eq. (4) }
$$

and percentage incidence $(I)$ values were transformed to arcsine incidence $\left(I_{\text {arcsin }}\right)$ :

$$
I_{\text {arcsin }}=\sin ^{-1}(\sqrt{ }(I / 100)) \quad \text { Eq. (5) }
$$

The incidence-severity relationship was determined by linear regression of $S_{\text {logit }}$ on $I_{\text {arcsin }}$, using GenStat version 11.

\section{RESULTS}

\section{Evaluation of BRAT}

Individual regressions of estimated versus actual \% botrytis severity (e.g. Figure $2 \mathrm{a}$ ) gave $\mathrm{R}^{2}$ values that ranged from $0.92-0.99$, showing that all assessors in all sessions were quite good at estimating \% severity. All the individual regression slope values were close to 1.0 (data not shown) and the mean slope did not vary significantly $(\mathrm{P}=0.149)$ in response to training (Table 1). Intercepts from individual regressions were significant for some assessors and sessions (data not shown). The mean intercept was negative in sessions 1 and 3 (Table 1), reflecting underestimation of severity at low severities when the assessment key was not used. Underestimation across all severities was greater in the pre-training (session 1), as shown by the significantly $(\mathrm{P}<0.05)$ greater negative ME (Table 1$)$. This shows that training with the assessment key improved accuracy. Precision also improved with training, as shown by the lower MAE in sessions 2 and 3, although session 2 was not significantly $(\mathrm{P}>0.05)$ different from session 1 (Table 1).

Table 1 Effect of level of training on accuracy and precision of estimated \% botrytis severity for eight users who each assessed 20 randomly selected images of diseased grape bunches. Intercepts and slopes are session means from individual regressions of estimated severity on actual severity. Mean error (ME) reflects overall accuracy and mean absolute error (MAE) reflects overall precision.

\begin{tabular}{lcccc}
\hline Session (level of training) & $\begin{array}{c}\text { Mean } \\
\text { Slope }\end{array}$ & $\begin{array}{c}\text { Mean } \\
\text { Intercept }^{1}\end{array}$ & $\begin{array}{c}\text { ME } \\
(\% \text { sev. })^{1}\end{array}$ & $\begin{array}{c}\text { MAE } \\
(\% \text { sev. })^{1}\end{array}$ \\
\hline 1. Pre-training (no assessment key) & 1.02 & $-5.0 \mathrm{a}$ & $-3.8 \mathrm{a}$ & $5.5 \mathrm{~b}$ \\
2. With assessment key & 0.97 & $1.1 \mathrm{~b}$ & $-0.3 \mathrm{~b}$ & $4.7 \mathrm{ab}$ \\
3. Post training (no assessment key) & 0.99 & $-1.5 \mathrm{ab}$ & $-1.7 \mathrm{~b}$ & $4.2 \mathrm{a}$ \\
P-value from ANOVA & 0.149 & 0.016 & $<0.001$ & 0.017 \\
\hline
\end{tabular}

${ }^{1}$ For variables where the ANOVA was significant $(\mathrm{P}<0.05)$, means followed by the same letter are not significantly different (Tukey's LSD). 

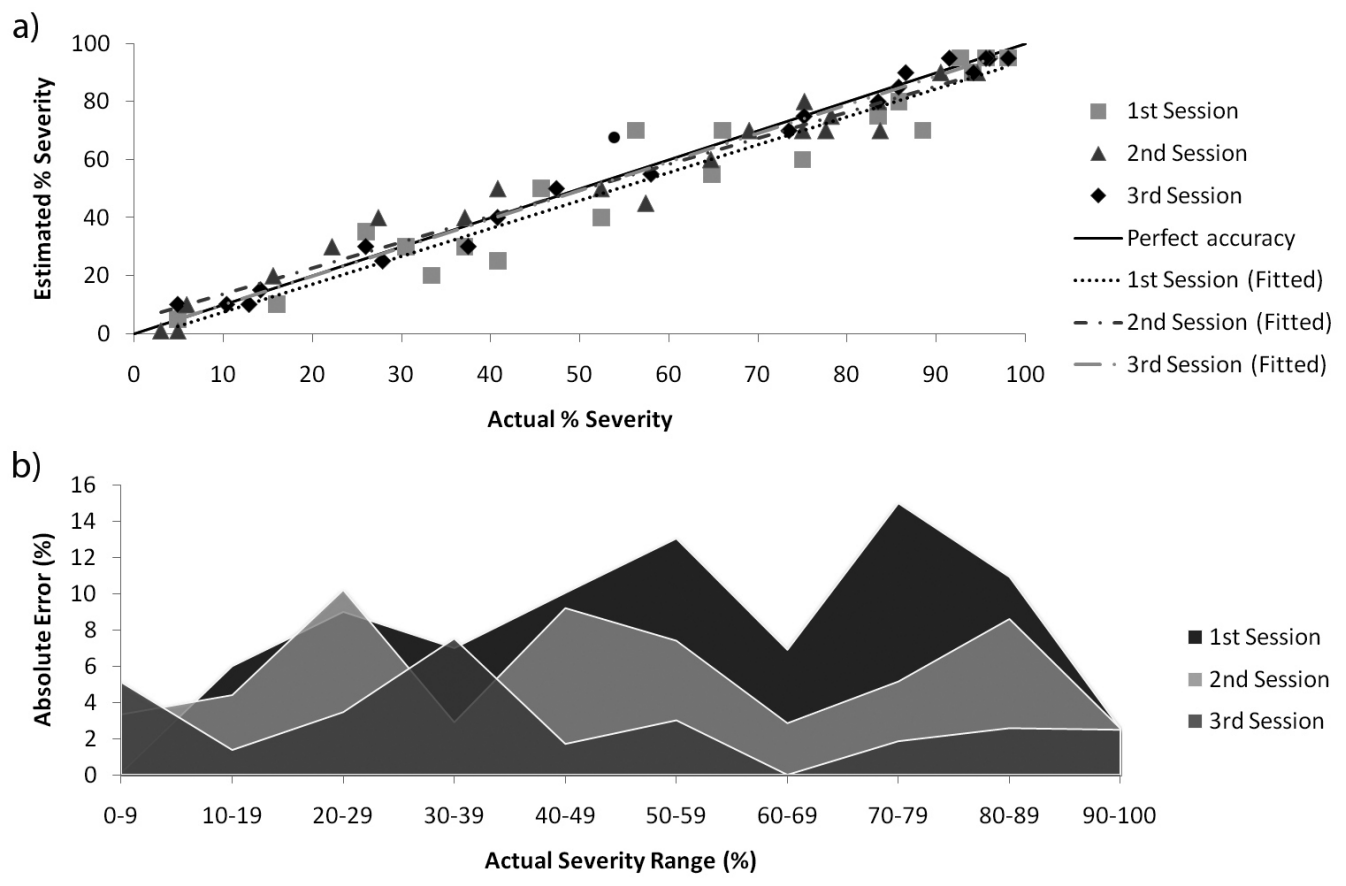

Figure 2 Bunch rot assessment trainer (BRAT) results from one user for three sessions (1. pre-training, 2. with assessment key and 3. post-training without assessment key), showing (a) Estimated versus actual severity with regression lines for session $1\left(y=0.962 x-2.105 ; \mathrm{R}^{2}=0.92\right)$, session $2(y=0.895 x$ $\left.+4.709 ; \mathrm{R}^{2}=0.96\right)$ and session $3\left(y=0.984 x+0.191 ; \mathrm{R}^{2}=0.99\right)$ and (b) Mean absolute error for each session in ten severity ranges.

BRAT results for one of the eight users, who improved their assessment accuracy through the use of the software, are shown in Figure 2. The regression output appeared to show little difference between the three sessions (Figure 2a), although the MAE for this single user dropped significantly $(\mathrm{P}<0.001)$ from $7.7 \%$ in the first session to $2.7 \%$ in the third session. This difference in MAE was more evident when botrytis severity was divided into severity categories (Figure 2b).

\section{Sample size}

Mean percentage difference from the CV of all available bunches (population $\mathrm{CV}$ ) was plotted against subset sample size (Figure 3). From this it was estimated that $\sim 100$ bunches were needed to obtain a reliable estimate of botrytis severity.

\section{Incidence-severity relationship}

A significant $(\mathrm{P}<0.001)$ relationship was found between arcsine-transformed botrytis incidence and logit-transformed botrytis severity, up to the maximum observed mean severity of $40 \%$ (Figure 4).

\section{DISCUSSION}

Use of the BRAT software gave an improvement in overall accuracy and precision of the eight users, even though all had previous experience with visual estimation methodologies. Greater improvements after training could be expected for users with no previous experience. These results are similar to those of Nutter (2001), who reported improvements in accuracy and precision for grapevine downy mildew severity assessment resulting from the use of the disease assessment training software, Severity.Pro. In addition to 
its use for training, the BRAT software could also be used to correct inaccuracy in previously collected botrytis assessment data. If an assessor was found to be consistently inaccurate (e.g. overestimating at low severities), the equation from the results graph could be used to adjust their severity values.

The precision of severity assessments in this study was described using MAE, whereas Nutter et al. (2006) used the standard error of the Y-estimate from the regression of estimated versus actual severity to describe precision. Both methods would serve a similar purpose when used to determine improvements in precision through training. The minimum sample size determined in this study can be used as a starting point for designing a botrytis sampling regime. Using Figure 3, the sample size could be altered to achieve a different level of precision. It is recommended that the bunch sample whose size has been determined using Figure 3 should be collected at random from an area of the vineyard with uniform risk of botrytis in relation to grape variety, fungicide regime, canopy management, microclimate, soil type and fertiliser. This method is only approximate and could underestimate the required sample size if the population $\mathrm{CV}$ was not determined from a sufficiently large sample.

To use the incidence-severity relationship for estimating botrytis severity $(S)$ from percentage incidence $(I)$, logit severity $\left(S_{\text {logit }}\right)$ is first calculated using arcsine-transformed incidence:

$$
S_{\text {logit }}=\mathrm{a}+\mathrm{b} \times \sin ^{-1}(\sqrt{ }(I / 100)), \quad \text { Eq. (6) }
$$

where $a$ is the intercept $(-6.254)$ and $b$ is the slope (0.062) of the I-S relationship (Figure 4). Then, $S_{\text {logit }}$ is back-transformed to $S$ :

$$
S=\left(100.2 /\left(1+\mathrm{e}^{-S_{\text {logit }}}\right)\right)-0.1 \quad \text { Eq. }(7)
$$

Severity estimation from incidence would be quicker to perform than a full severity assessment of individual bunches and could be useful where

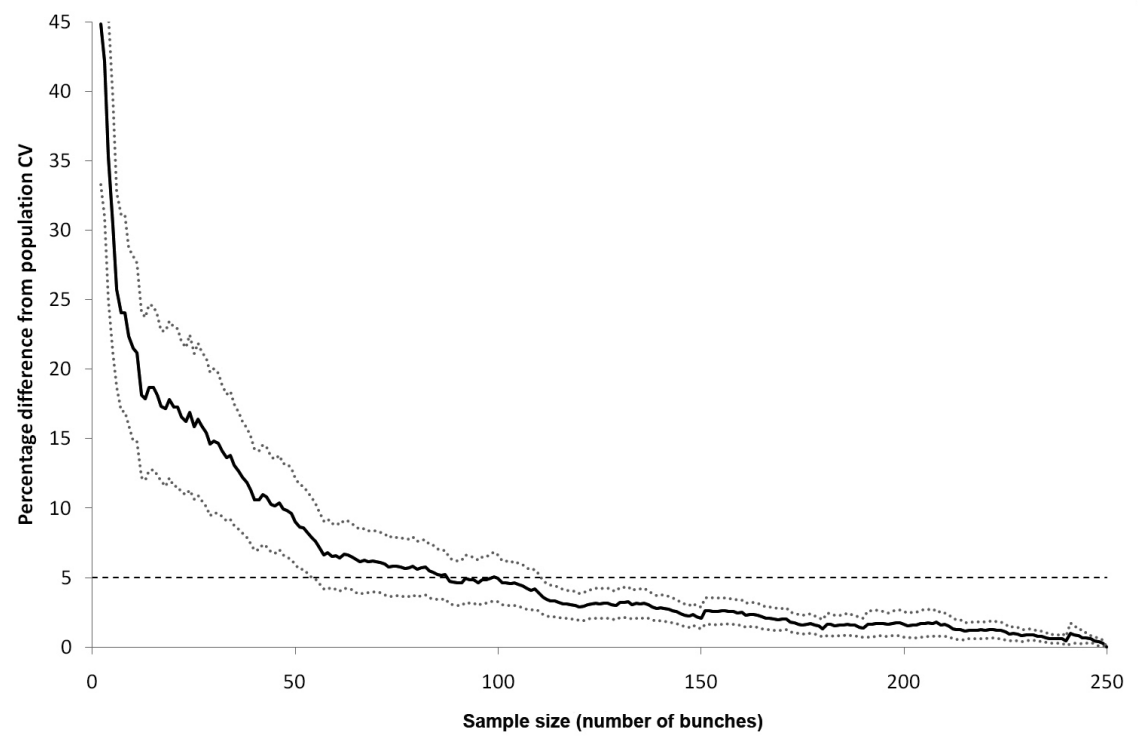

Figure 3 Percentage difference between the coefficient of variation (CV) for mean botrytis \% severity of a sample and the CV of all 250 available bunches (population CV). Samples of increasing size were taken from random subsets of severity data collected at five vineyards in the 2008/2009 season. The solid line is the mean of all random subsets of a particular sample size, dotted lines are the upper and lower confidence intervals $(\alpha=0.05)$ and the dashed line indicates a $5 \%$ difference threshold from the population $\mathrm{CV}$. 


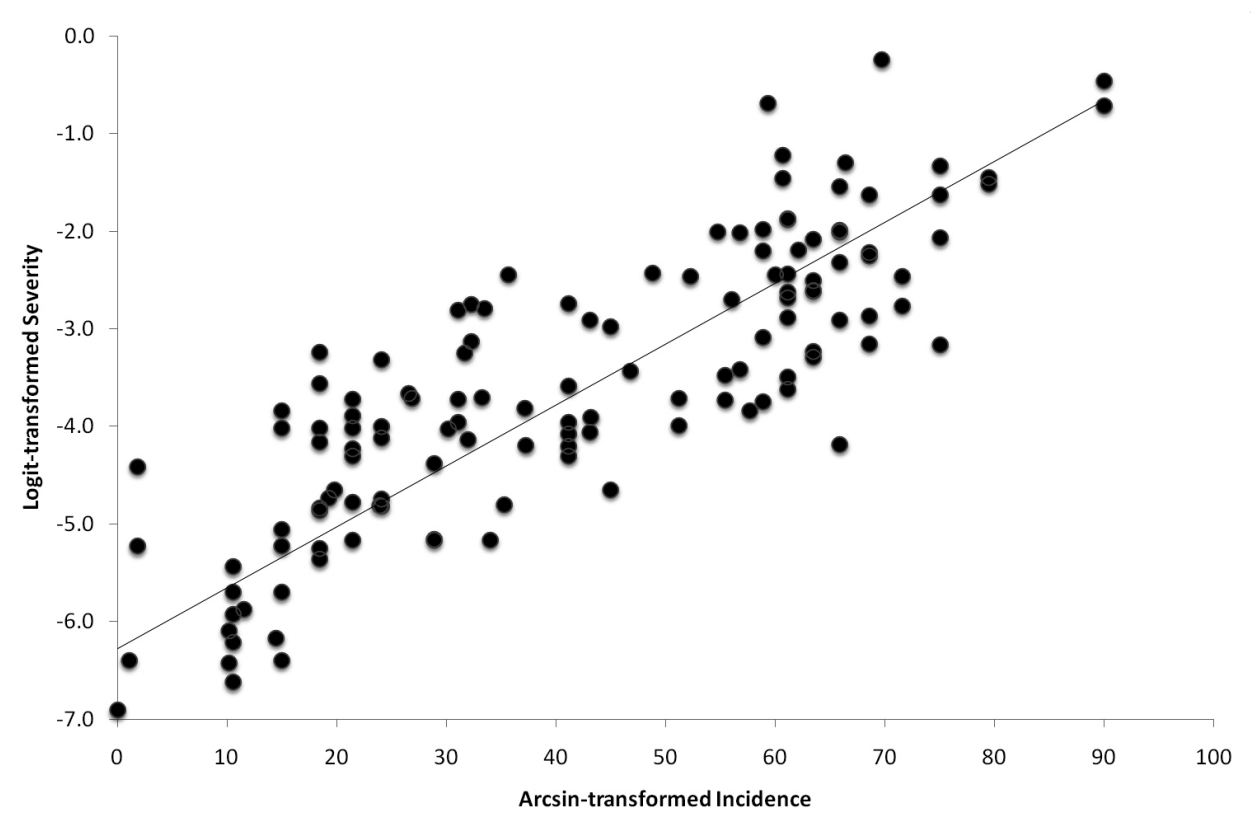

Figure 4 Relationship between botrytis severity on grape bunches and botrytis incidence $(y=0.062 x$ $\left.-6.254 ; \mathrm{R}^{2}=0.79 ; \mathrm{P}<0.001\right)$ derived from the incidence and mean severity from 126 samples of $30-50$ bunches. Severity data have been logit-transformed and incidence data have been arcsin-transformed to linearise the relationship.

rapid estimation of botrytis is required, e.g. to detect the time of onset of a botrytis epidemic in a vineyard. However, to accurately determine the degree to which a grape crop is affected by botrytis, e.g. for winery information at harvest, a severity assessment of individual bunches using the severity key would be required.

With the aid of the tools described in this paper, it should be possible to obtain reliable botrytis severity data for use by viticulturalists and wine makers. These tools, when used together with the methods for botrytis epidemic monitoring described by Beresford et al. (2008), will provide high quality data for inputs into decision support models and for research into botrytis epidemiology and control. It is important to note that tools such as the botrytis severity assessment key and BRAT software are not only intended for the training of assessors with little to no experience; they may also be used to improve or refresh the skills of more experienced assessors. Additionally, these tools may be applicable to bunch rots caused by other fungi (e.g. Botryosphaeria spp.) and bacteria (e.g. Acetobacter spp.) that produce similar symptoms to botrytis.

\section{ACKNOWLEDGEMENTS}

Development of the Bunch Rot Assessment Trainer (BRAT) was funded by the New Zealand Foundation for Research, Science and Technology (C06X0810). We thank Dion Mundy, Kerry Everett, Kwang Soo Kim, Michelle Beresford, Pia Rheinländer and Reiny Scheper (Plant \& Food Research) for testing the BRAT software. The sample size and incidence-severity relationship data were collected by Peter Wood, Tracy Taylor, Dion Mundy and Kwang Soo Kim (Plant \& Food Research), Justin Direen (TIAR) and Jacky Edwards and David Riches (Department of 
Primary Industries, VIC, Australia) in projects funded by New Zealand Winegrowers and the Grape and Wine Research and Development Corporation (Australia).

\section{REFERENCES}

Beresford RM, Evans KJ, Wood PN, Mundy DC 2006. Disease assessment and epidemic monitoring methodology for bunch rot (Botrytis cinerea) in grapevines. New Zealand Plant Protection 59: 355-360.

James WC 1974. Assessment of plant diseases and losses. Annual Review of Phytopathology 12: 27-48.

Kranz J 1988. Measuring Plant Disease. In: Kranz J, Rotem J ed. Experimental techniques in plant disease epidemiology. Springer-Verlag Heidelberg, Berlin, Germany. Pp. 35-50.

Nair NG, Hill GK 1992. Bunch rot of grapes caused by Botrytis cinerea. In: Kumar J, Chaube HS, Singh US, Mukhopadhyay AN ed. Plant diseases of international importance. v. III: Diseases of fruit crops. Prentice-Hall,
Inc., Englewood Cliffs, NJ, USA. Pp. 147-169. Nutter FW 2001. Disease assessment terms and concepts. In: Maloy OC, Murray TD ed. Encyclopedia of Plant Pathology. John Wiley and Sons, New York. Pp. 312-323.

Nutter FW, Esker PD, Netto RAC 2006. Disease assessment concepts and the advancements made in improving the accuracy and precision of plant disease data. European Journal of Plant Pathology 115 (1): 95-103.

Seem RC 1984. Disease incidence and severity relationships. Annual Review of Phytopathology 22 (1): 133-150. 